\title{
Initial experience with the coaxial dual-lumen Ascent balloon catheter for wide-neck aneurysm coil embolization
}

\author{
Marc A. Lazzaro' ${ }^{1}$ Ziad Darkhabani ${ }^{1}$, Osama O. Zaidat ${ }^{1,2,3}$ and Brian-Fred M. Fitzsimmons , $^{1,2,3}$ * \\ ${ }^{1}$ Department of Neurology, Froedtert Hospital and Medical College of Wisconsin, Milwaukee, WI, USA \\ 2 Department of Neurosurgery, Froedtert Hospital and Medical College of Wisconsin, Milwaukee, WI, USA \\ ${ }^{3}$ Department of Radiology, Froedtert Hospital and Medical College of Wisconsin, Milwaukee, WI, USA
}

\section{Edited by:}

Shakir Husain Hakim, Max Institute of

Neurosciences, India

Reviewed by:

Johanna Fifi, Albert Einstein College

of Medicine, USA

Mohamed Elmahdy, Cairo University,

Egypt

*Correspondence:

Brian-Fred M. Fitzsimmons, Department of Neurology, Froedtert Hospital and Medical College of Wisconsin, 9200 West Wisconsin

Avenue, Milwaukee, WI 53226, USA e-mail: bfitzsim@mcw.edu
Introduction: Techniques for coil embolization of wide-neck cerebral aneurysms include the use of stents and temporary occlusion with compliant non-detachable balloons to safely allow dense packing of the aneurysm lumen with detachable coils. We describe the use of a new balloon device for assisting in wide-neck aneurysm coil treatment. Methods: A single institution neuroendovascular database was accessed to identify cases in which the Ascent balloon (Codman Neurovascular, Raynham, MA, USA) was used for aneurysm coil embolization. Clinical, demographic, and angiographic data were obtained through chart review. Results: Eleven cerebral aneurysm cases were treated using the Ascent balloon during the first 12-month period that the new device was available at our institution. Three of the patients presented with ruptured aneurysms. All aneurysms were large (maximum diameter $6 \mathrm{~mm}$ or greater), with an average maximum diameter of $9.4 \mathrm{~mm}$, and an average neck diameter of $5.5 \mathrm{~mm}$. Complete occlusion with coil embolization (Raymond class l) was achieved in all cases. The Ascent balloon was successfully positioned across the neck of the aneurysm in nine patients. Conclusion: This initial experience demonstrates the feasibility and immediate outcomes of the coaxial dual-lumen design Ascent balloon catheter used as an assistive device in coil embolization of wide-neck cerebral aneurysms. This device contributes to the growing number of assistive devices for the treatment of complex cerebral aneurysms.

Keywords: aneurysm embolization, ascent, balloon remodeling, coil

\section{INTRODUCTION}

Advancements in endovascular devices and techniques for aneurysm coil embolization continue to expand the ability to treat complex aneurysms. Wide-neck aneurysms often require assistive devices and techniques to prevent coil prolapse into the parent artery during coil placement and to allow dense coil packing. Temporary parent artery occlusion, or "balloon remodeling," has become a well-described assistive technique for coil embolization of wide-neck aneurysms (Moret et al., 1997; Lefkowitz et al., 1999; Aletich et al., 2000). In this technique, a non-detachable balloon is used for temporary vessel occlusion across the aneurysm neck; serving to stabilize the microcatheter, direct coil placement, and prevent coil prolapse, which assists in safer and more dense coil packing. Early reports of the balloon remodeling technique used less compliant balloons that were either oval or round in shape, characteristics that limited the effectiveness in wide-neck aneurysms at bifurcations (Moret et al., 1997). Newer generation more compliant balloons allow for greater conformability to arterial bifurcations and asymmetric vessels, which theoretically provides a more secure seal at the aneurysm neck. Until recently, only two over-the-wire, single-lumen compliant non-detachable balloons have been in use for this technique, the HyperForm and HyperGlide balloons (ev3 Neurovascular, Plymouth, MN, USA; Baldi et al., 2003). The Ascent balloon (Codman Neurovascular, Raynham, MA, USA) is a newer compliant balloon for temporary occlusion in aneurysm coil embolization with a coaxial duallumen design that allows delivery over an exchange wire. We report a series of early experience with this new device.

\section{MATERIALS AND METHODS}

A search of our neuroendovascular database was performed to identify cases in which the Ascent balloon (Codman Neurovascular, Raynham, MA, USA) was used for cerebral aneurysm embolization during the first 12-month period that the new balloon was available at our institution. Clinical, demographic, and procedural data were obtained from charts and relevant angiographic images were reviewed.

Procedures were performed with patients under general anesthesia. A transfemoral arterial approach was used. Per protocol, patients were systemically heparinized to maintain an ACT level of 250-300 s, and a coaxial system including a 6F 070 Neuron guide catheter (Penumbra, Inc., San Leandro, CA, USA) was used. Images were obtained with biplane projections and a 3-D rotational digital subtraction angiogram was obtained for each patient. Working views were obtained after review of the 3-D rotational angiogram. The Ascent balloon was prepped following manufacturer recommendations. A $0.014^{\prime \prime}$ microwire was used to advance the Ascent balloon catheter to the aneurysm neck. The aneurysm lumen was accessed with a microcatheter. Intermittent temporary balloon inflation was performed at the time of coil placement 
(Figures 1-4). Final angiographic runs were performed prior to completion of each case to evaluate aneurysm residual, parent artery patency, and thrombotic or dissection complications.

\section{RESULTS}

Eleven patients underwent balloon-assisted coil embolization of wide-neck aneurysms with the Ascent balloon system. Patient and aneurysm characteristics are summarized in the table (Table 1). There were nine females $(82 \%)$. Three cases involved ruptured aneurysms. All aneurysms were large (maximum diameter $6 \mathrm{~mm}$ or greater) with a range from 6 to $15 \mathrm{~mm}$ in maximum diameter. The average aneurysm neck length was $5.5 \mathrm{~mm}$. Ascent balloon sizes ranged from $4 \mathrm{~mm} \times 7 \mathrm{~mm}$ to $4 \mathrm{~mm} \times 15 \mathrm{~mm}$. The Ascent balloon was successfully delivered to the aneurysm neck in nine

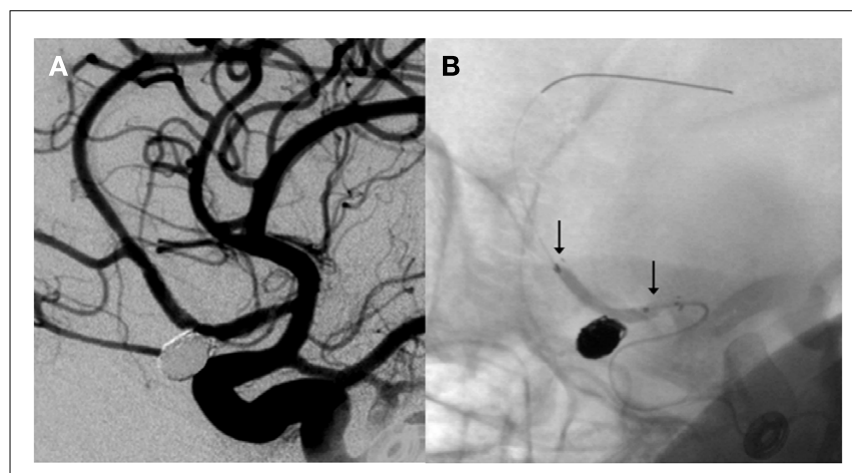

FIGURE 1 | Right internal carotid artery injection angiogram demonstrates coil embolization of a right anterior cerebral artery aneurysm (A) using balloon remodeling with the Ascent balloon [arrows, (B)].

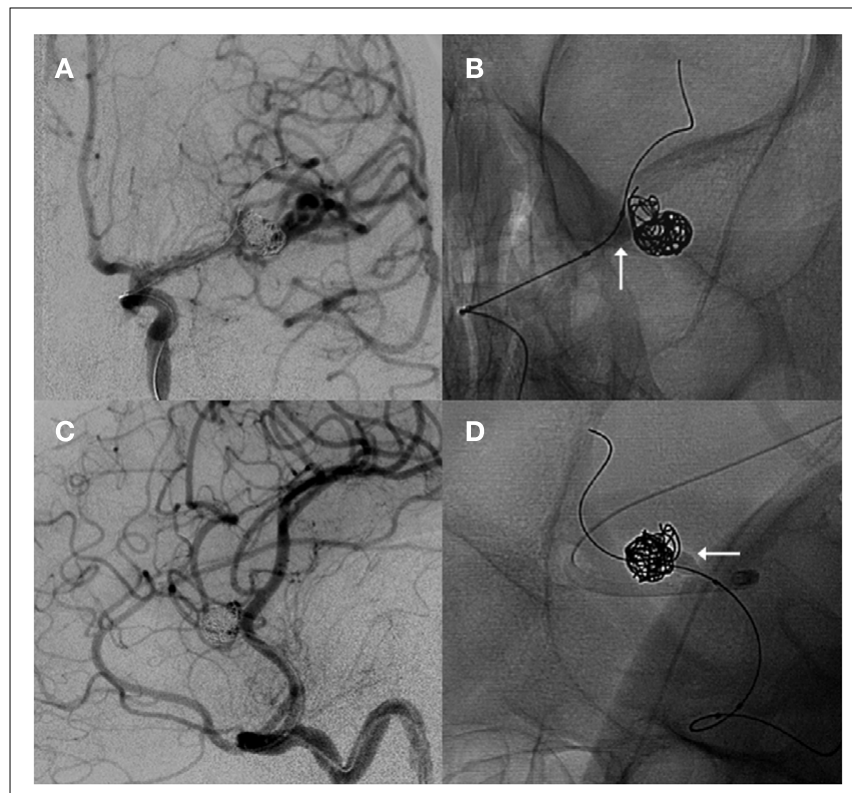

FIGURE 2 | Anteroposterior (A) and lateral oblique (C) left internal carotid artery injection angiogram projections demonstrate coil embolization of a left middle cerebral artery aneurysm with balloon remodeling using the Ascent balloon [arrows, (B,D)]. cases and was unable to be successfully used in two cases. There was no microcatheter displacement from balloon inflation. No parent artery rupture or dissection occurred in the present series. Coil embolization was complete, Raymond class I, in all cases.

Transient thrombus was identified distal to the site of balloon placement in the parent artery of one of the ruptured aneurysm cases (Case 1), which resolved completely after antiplatelet administration and without clinical sequelae.

In case 2, packing of the broad neck aneurysm resulted in temporary coil herniation into the right A2 segment producing a reduction in antegrade flow due to adherent thrombus. Abciximab was administered $(15 \mathrm{mg})$ intravenously followed by placement of a Neuroform 3 stent across the aneurysm neck with successful repositioning of all coil loops into the aneurysm and improved

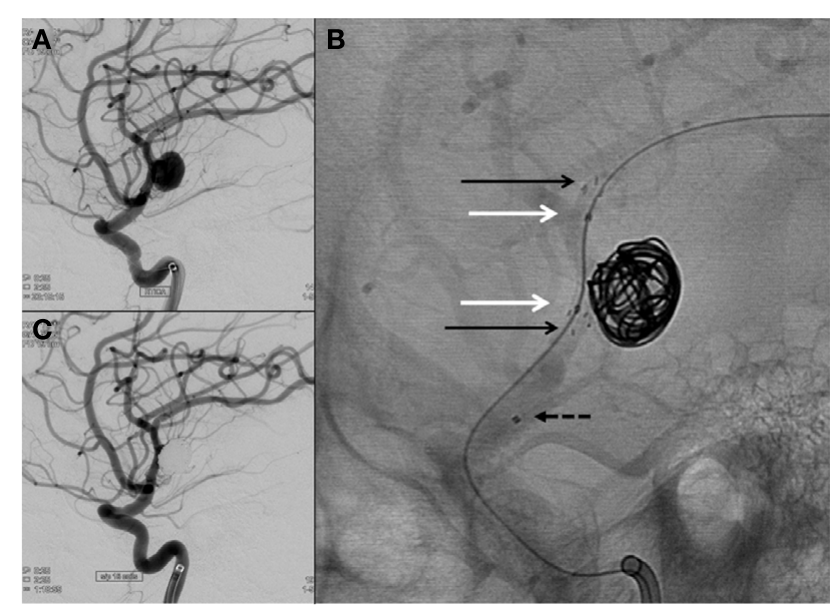

FIGURE 3 | A wide-neck blowout morphology right MCA aneurysm (A) was treated using a balloon-in-stent technique. An Enterprise stent was implanted across the aneurysm neck [(B), black arrows] and an Ascent balloon was intermittently inflated across the neck (white arrows) for balloon remodeling. The microcatheter position was secured with the balloon using a jailing technique (proximal microcatheter marker shown by dashed arrow). Complete occlusion of the aneurysm was achieved with adequately dense coil packing (C).

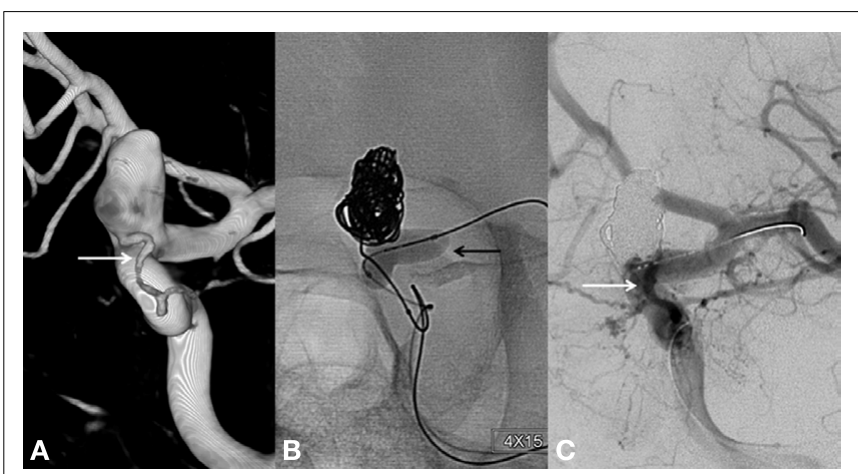

FIGURE 4 | A large wide-neck paraophthalmic artery aneurysm with the ophthalmic artery arising from the aneurysm neck (A) was treated with Ascent balloon remodeling [(B), black arrow]. Complete aneurysm occlusion was achieved with preservation of the ophthalmic artery [(C), white arrow]. 


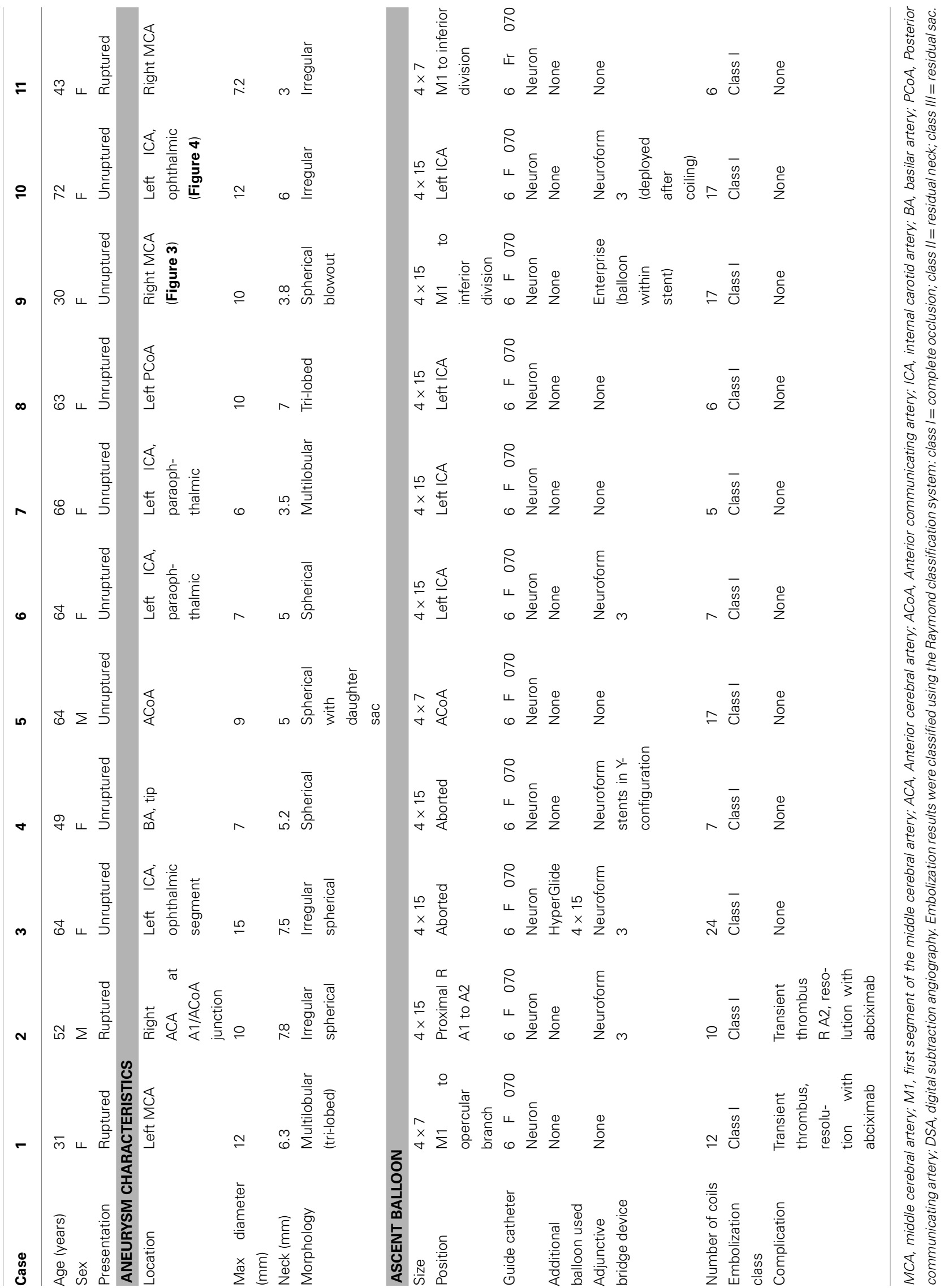


antegrade flow. A partially inflated Ascent balloon was then placed within the stent for additional support.

Use of the Ascent balloon was unsuccessful in two cases. The inability to track the Ascent balloon around the ophthalmic segment in case 3 prohibited adequate delivery, while a HyperGlide balloon allowed successful positioning at the aneurysm neck. In case 4 , multiple attempts were made to deliver the Ascent balloon, however tortuosity prohibited safe positioning of the balloon microcatheter. An alternative compliant balloon was also unsuccessfully attempted, and ultimately Y-configuration stenting was performed to secure a neck bridge.

\section{DISCUSSION}

We report our initial experience with a coaxial dual-lumen Ascent balloon catheter system for the remodeling technique in wideneck aneurysm treatment. This is the largest series describing the use of this device for wide-neck aneurysm treatment. This report contributes to the growing body of literature describing novel techniques and new devices that expand the possibilities of endovascular treatment of complex aneurysms.

The purpose of temporary parent artery occlusion in aneurysm coil embolization is to prevent coil prolapse, improve coil positioning, and allow for greater density packing. A recent meta-analysis suggested that balloon-assisted coil embolization leads to greater initial occlusion rates and more durable occlusion when compared to unassisted coil embolization (Shapiro et al., 2008). Additionally, the balloon occlusion technique avoids permanent device implantation as compared to stent-assisted embolization techniques, which is of considerable benefit in treatment of ruptured aneurysms when antithrombotic therapy poses risk of hemorrhagic complication. Complete aneurysm occlusion was achieved in all aneurysms in this series.

Aneurysm size was large in our series, ranging from 6 to $15 \mathrm{~mm}$. The average neck size was also large, ranging from 5.0 to $7.8 \mathrm{~mm}$. These characteristics are important for balloon performance as larger neck size may pose greater challenge in initial balloon positioning and maintaining placement during repeated inflations.

Aneurysm locations varied, providing a reasonably welldistributed sample. The patient population was relatively young (average age 54 years) and vessel tortuosity was moderate. As with any device, navigation of tortuous vessels will hinder performance and this was possibly the cause of inability to safely position the Ascent balloon in cases 3 and 4. Improved distal support through use of a distal access catheter may reduce this limitation.

A unique characteristic of the Ascent balloon system is the duallumen design that allows delivery of the balloon catheter over a variety of microwires, allowing for flexibility in the choice of wires. Performance with use of different wires is not known and further experience may identify specific wires that provide an optimal pairing. This design may be of potential usefulness in the event of artery rupture. In the present series, this new device allowed use of an exchange-length wire, which is not feasible with other compliant aneurysm balloon catheters. Moreover, the over-the-wire devices do not allow for saline perfusion around the microwire or removing the wire to switch to a different microwire while leaving the balloon catheter in the body. Occlusion of the balloon holes with blood clots is another potential drawback of the over-the-wire balloons preventing full deflation on occasion.

The dual-lumen design allows for delivery of the Ascent balloon over an exchange wire, which can be useful in the balloon-in-stent remodeling technique (Figure 3). Following delivery of a stent, the exchange wire can remain in position, securing distal access, and allow for advancement of the dual-lumen balloon to the aneurysm neck. This eliminates the need to re-cross the stent.

The ability of a device to track well within the cerebrovasculature, or the "trackability," is an important characteristic of any neurointerventional device. This concept involves factors that affect the ability of a device to travel the course of a vessel, which include the inherent characteristics of a device, such as the flexibility and responsiveness to proximal manipulation, as well as the behavior of the device as a result of interaction with the surrounding vessel, such as the ability to glide. The Ascent balloon was not successfully used in two of our cases due to the inability to advance the balloon to the target lesion, which may have been from the device trackability in a tortuous cerebrovascular anatomy. The trackability may be related to interaction of the device over an exchange wire, a characteristic that may be important in wire selection. The trackability may also be affected by stiffness, a characteristic that may be theoretically increased due to the dual-lumen design.

Repeated balloon inflation may lead to unintended movement of the adjacent microcatheter resulting in loss of aneurysm lumen access, balloon migration, and parent artery rupture. Five cases required delivery of 10 or more coils, suggesting balloon positioning and access is well maintained throughout the technique of intermittent balloon inflation.

Complications from balloon remodeling primarily include thromboembolism, which range from about 2 to $8 \%$, and parent artery perforation, which is under $2 \%$ in large series (Shapiro et al., 2008; Cekirge et al., 2011). A transient clot formation was noted in case 1 distal to the site of balloon placement in the parent artery. This thrombus resolved during abciximab administration and the patient experienced no clinical sequelae. The simultaneous use of two microcatheters and resulting compromised perfusion in small arteries may theoretically contribute to thrombotic risk in this technique, however there has been no evidence of an increased risk of thromboembolic complications in the balloon-assisted technique compared to unassisted coil embolization (Shapiro et al., 2008). Follow-up outcome measures include occlusion durability, which is about $72 \%$ in large series (Moret et al., 1997; Shapiro et al., 2008).

Limitations of this report include the retrospective design and small sample size. Additionally, selection bias exists among operators and the decision to use one of three non-detachable compliant balloons available at our institution.

\section{CONCLUSION}

This initial experience demonstrates the feasibility and immediate results of a coaxial dual-lumen balloon catheter as an assistive device in coil embolization of wide-neck cerebral aneurysms. These findings are encouraging with expansion of the growing armamentarium of endovascular devices and techniques for the treatment of complex cerebral aneurysms. Larger series are needed to further evaluate this device for efficacy and long-term outcomes. 


\section{REFERENCES}

Aletich, V. A., Debrun, G. M., Misra, M., Charbel, F., and Ausman, J. I. (2000). The remodeling technique of balloon-assisted Guglielmi detachable coil placement in wide-necked aneurysms: experience at the University of Illinois at Chicago. $J$. Neurosurg. 93, 388-396.

Baldi, S., Mounayer, C., Piotin, M., Spelle, L., and Moret, J. (2003). Balloon-assisted coil placement in wide-neck bifurcation aneurysms by use of a new, compliant balloon microcatheter. AJNR Am. J. Neuroradiol. 24, 1222-1225.

Cekirge, H. S., Yavuz, K., Geyik, S., and Saatci, I. (2011). HyperForm balloon remodeling in the endovascular treatment of anterior cerebral, middle cerebral, and anterior communicating artery aneurysms: clinical and angiographic follow-up results in 800 consecutive patients. J. Neurosurg. 114, 944-953.

Lefkowitz, M. A., Gobin, Y. P., Akiba, Y., Duckwiler, G. R., Murayama, Y., Guglielmi, G., Martin, N. A., and Vinuela, F. (1999). Ballonnassisted Guglielmi detachable coiling of wide-necked aneurysma: part II - clinical results. Neurosurgery 45, 531-537; discussion 537-538.

Moret, J., Cognard, C., Weill, A., Castaings, L., and Rey, A. (1997). The "remodelling technique" in the treatment of wide neck intracranial aneurysms. Angiographic results and clinical follow-up in 56 cases. Interv. Neuroradiol. 3, 21-35.

Shapiro, M., Babb, J., Becske, T., and Nelson, P. K. (2008). Safety and efficacy of adjunctive balloon remodeling during endovascular treatment of intracranial aneurysms: a literature review. AJNR Am. J. Neuroradiol. 29, 1777-1781.

Conflict of Interest Statement: The authors declare that the research was conducted in the absence of any commercial or financial relationships that could be construed as a potential conflict of interest.

Received: 20 June 2011; accepted: 02 August 2011; published online: 19 August 2011.
Citation: Lazzaro MA, Darkhabani $Z$, Zaidat $\mathrm{OO}$ and Fitzsimmons B-FM (2011) Initial experience with the coaxial dual-lumen Ascent balloon catheter for wide-neck aneurysm coil embolization. Front. Neur. 2:52. doi: 10.3389/fneur.2011. 00052

This article was submitted to Frontiers in Endovascular and Interventional Neurology, a specialty of Frontiers in Neurology. Copyright (ङ 2011 Lazzaro, Darkhabani, Zaidat and Fitzsimmons. This is an open-access article subject to a nonexclusive license between the authors and Frontiers Media SA, which permits use, distribution and reproduction in other forums, provided the original authors and source are credited and other Frontiers conditions are complied with. 